\title{
Modulation of Surface Bonding Topology: Oxygen Bridges on OH- terminated InP (001)
}

\author{
Xueqiang Zhang, ${ }^{1,2 *}$ Tuan Anh Pham, ${ }^{3 * \$}$ Tadashi Ogitsu, ${ }^{3}$ Brandon C. Wood, ${ }^{3}$ Sylwia \\ Ptasinska $^{1,4^{*}}$ \\ ${ }^{1}$ Radiation Laboratory, University of Notre Dame, Notre Dame, IN 46556, USA \\ ${ }^{2}$ Department of Chemistry and Biochemistry, University of Notre Dame, Notre Dame, IN 46556, USA \\ ${ }^{3}$ Quantum Simulations Group, Lawrence Livermore National Laboratory, Livermore, CA \\ 94551, United States \\ ${ }^{4}$ Department of Physics, University of Notre Dame, Notre Dame, IN 46556, USA
}

Supplementary information

*Author to whom correspondence should be addressed.

${ }^{\$}$ These authors contributed equally

${ }^{+}$Current address: 1 Cyclotron Rd., Advanced Light Source, Lawrence Berkeley National Laboratory, Berkeley, CA 94820, USA

Tel: +1-574-631-4506. E-mail: xzhang10@alumni.nd.edu; pham16@1lnl.gov;

Sylwia.Ptasinska.1@nd.edu.

Postal Address: Radiation Laboratory, University of Notre Dame, Notre Dame, IN 46556 USA. 


\section{Experimental setup, procedure and data analysis}

All photoemission spectra were recorded using a custom-built ambient pressure X-ray photoelectron spectrometer (APXPS, SPECS Surface Nano Analysis GmbH, Germany). The spectrometer consisted of three major vacuum chambers, i.e., load-lock, preparation, and analysis chambers. The analysis chamber with a base pressure of $5 \times 10^{-10}$ mbar was equipped with an $\mathrm{Al}$ Ka X-ray tube $(1486.7 \mathrm{eV})$ coupled to a Micro-FOCUS 600 monochromator (XR-MF). Inside the analysis chamber a minimized reaction cell $\left(15 \mathrm{~cm}^{3}\right)$ was attached to a differentially-pumped, electrostatic lens system and a PHOIBOS 150 hemispherical energy analyzer. An X-ray source anode voltage of $15 \mathrm{kV}$ and an emission current of $6.7 \mathrm{~mA}$ were used to achieve a power of 001 W. The X-ray beam was transmitted through a silicon nitride window located on the side wall of the reaction cell, and the incident beam impacted a sample at a fixed angle of $54.7^{\circ}$ to the surface normal. Detailed descriptions of the experimental setup have been reported in our previous work. ${ }^{1,2}$

The photoemission energy scales were referenced to the Fermi edge of clean $\mathrm{Au}(111)$ and were recalibrated on weekly basis. The binding energy (BE) scales for the photoemission spectra were calibrated using the $\mathrm{P} 2 \mathrm{p}_{3 / 2}$ peak, which corresponds to the P-In bond with a BE of $128.8 \mathrm{eV}^{3-6}$ Note that the vigorous chemical changes at high temperatures (>673 K) lead to complication of the InP (001) surface chemistry, and the BE calibrations were performed by assuming the peak maximums of $\mathrm{P} 2 \mathrm{p}_{3 / 2}$ and In $4 \mathrm{~d}_{5 / 2}$ still represent P-In bond. All spectra were fitted with a multiple Gaussian/Lorentzian peak combination using SpecsLab2 and CasaXPS software. A flexibility in the range of less than $0.1 \mathrm{eV}$ for the $\mathrm{BE}$ and full width at half-maximum (FWHM) were used in our peak-fitting analysis. Note that, using BEs from our direct spectroscopic simulations, much more precise spectral fittings and peak assignments were achieved. The FWHM of the Au 4f $7 / 2$ 
peak was $0.5 \mathrm{eV}$ at a pass energy of $20 \mathrm{eV}$, while the inherent lifetime broadening of this peak has been reported to be $\sim 0.3 \mathrm{eV} .^{7}$

An InP (001) crystal was purchased from Wafer Technology, UK (undoped, $\pm 0.1^{\circ}$, Mobility $\geq$ $\left.4200 \mathrm{~cm}^{2} \mathrm{~V}^{-1} \mathrm{~s}^{-1}\right)$. The InP (001) crystal was cleaned in the preparation chamber that was directly connected to the analysis chamber via a gate valve. We adopted the cleaning method reported by Sung et al., ${ }^{8}$ in which a low energy $(30 \mathrm{eV}) \mathrm{Ar}^{+}$beam was applied for $10 \mathrm{~min}$ with an angle of $45^{\circ}$ with respect to the InP (001) surface, followed by annealing at $573 \mathrm{~K}$ for $10 \mathrm{~min}$. The crystal temperature was monitored by a K-type (chromel-alumel) thermocouple inserted between the InP (001) substrate and a molybdenum sample holder. No $\mathrm{Ar}^{+}$ion implantation was observed after the pretreatment. Prior to measurement, the thermocouple was calibrated using a Lumasense Pyrometer. Ultra-pure water was degassed by multiple freeze-pump-thaw cycles prior to use. The C 1s spectra were recorded frequently throughout the experimental procedures with no carbon contamination detected. Note that, the reaction cell was retracted and thoroughly cleaned prior to the in-situ experiments using ethanol and acetone. After pumping down, the sample stage, along with most part of the reaction cell, was heated to $773 \mathrm{~K}$ under continuous flushing of argon and $\mathrm{O}_{2}$ to burn off adventitious contaminations.

We performed three types of photoemission experiments in which the InP (001) crystals were subjected to different conditions. 1) An isothermal study was conducted at room temperature (RT) and the $\mathrm{H}_{2} \mathrm{O}$ pressure was adjusted from ultra-high vacuum (UHV, $5 \times 10^{-10} \mathrm{mbar}$ ) to 5 mbar. In this experiment, we recorded pressure-dependent photoemission spectra at UHV, 0.005, 0.05, 0.5, and 5 mbar after the $\mathrm{H}_{2} \mathrm{O}$ pressure was stabilized ( 20 min); 2) After $\mathrm{H}_{2} \mathrm{O}$ exposure, the reaction cell was vacuum evacuated, and then the InP (001) was annealed from RT to $723 \mathrm{~K}$; 3) An isobaric study was conducted in which the $\mathrm{H}_{2} \mathrm{O}$ pressure was constant at $0.1 \mathrm{mbar}$ and the temperature was 
varied, ranging from RT to $873 \mathrm{~K}$. In this experiment, we recorded temperature-dependent photoemission spectra at $373,474,573,673,773$, and $873 \mathrm{~K}$. During the temperature-dependent investigations, photoemission spectra were recorded after heating the crystal at a rate of $5 \mathrm{~K} / \mathrm{min}$, followed by $20 \mathrm{~min}$ of stabilization time. For a typical set of spectra, the acquisition time was within $0.5 \mathrm{~h}$, whereas in the higher pressure studies, i.e., > 1 mbar, the acquisition time was extended up to $1 \mathrm{~h}$ since the photoemission signal was decreased due to the greater scattering of photoelectrons. No time-dependent chemical composition changes were observed in the pressuredependent study, even with prolonged exposures up to several hours. However, in the temperaturedependent study, time-dependent variations in the intensities of photoemission spectra were observed due to ongoing reactions, i.e., up to $5 \%$ for temperatures below $673 \mathrm{~K}$ and $\sim 10-15 \%$ for 673-873 K. These variations were related to quantities and generally do not lead to new types of species forming on the InP (001) surface.

\section{Estimation of the thickness of surface oxide/phosphate:}

Assuming that homogeneous and uniform oxide/phosphate layers were formed on the $\mathrm{InP}(001)$ surface, the thickness of the atop layer $(d)$ can be estimated from the integrated peak intensities of In $3 \mathrm{~d} 5 / 2:^{3,9-11}$

$$
I=I^{o x} \exp \left(\frac{-d}{\lambda \cdot \sin (\alpha)}\right)
$$

where $\lambda$ is the inelastic mean free path (IMFP) of photoelectrons, i.e., $\sim 26$ and $\sim 34 \AA$ for photoelectrons from the In $3 \mathrm{~d}_{5 / 2}$ and In $4 \mathrm{~d}_{7 / 2}$ electronic states, respectively. ${ }^{12}$ The integrated photoelectron peak intensities, $I$ and $I^{o x}$, were corresponded to the InP surface with and without atop films, respectively. The value of $\sin (\alpha)(\alpha$ is the take-off angle of photoelectrons with respect to a surface) was 1 for our experimental setup. The thickness of surface oxides/phosphate can be 
estimated by dividing the atop layer thickness by the height of one atomic layer $(1.5 \AA$ for indium phosphide). ${ }^{3}$ Therefore, $8 \%$ and $60 \%$ of the shoulder area of the In $3 \mathrm{~d} 5 / 2$ photoemission spectra correspond to thickness of atop layers of $1.3 \mathrm{ML}$ and $15 \mathrm{ML}$, respectively

\section{First-principles simulations and core-binding energy calculations}

To aid the experimental study and capture the structure and chemistry of the InP photoelectrode under realistic conditions, we considered a fully hydroxylated In-rich (001) surface in direct contact with liquid water. This surface can be considered as an appropriate model for studying the equilibrated interface of $\mathrm{InP}(001)$ with neutral-pH water, as our prior simulations suggested that the surface oxide would lead to dense surface hydroxylation on the InP surface. ${ }^{13,14}$ Here, the hydroxylated interfaces with water was generated using seven semiconductor layers oriented along (001) with top and bottom layers identically terminated. The $\operatorname{InP}(001) /$ water interfacial model was used as inputs in Car-Parrinello molecular dynamics simulations, which were carried out within the canonical NVT ensemble using the Quantum-ESPRESSO code. ${ }^{15}$ The simulations were carried out at $400 \mathrm{~K}$, since the elevated temperature has been shown to be necessary to reproduce the structural properties of ambient liquid water. A fictitious electronic mass of 700 a.u. and a time step of 12 a.u. were used, and deuterium was substituted for hydrogen to permit the larger values. Configurations employed for electronic structure calculations were extracted from production runs of about $20 \mathrm{ps}$, after equilibration runs of $3.5 \mathrm{ps}$.

We used scalar-relativistic ultrasoft pseudopotentials with semicore $d$ states included in the valence descriptions for In. Kinetic energy cutoffs of 30 and 300 Ry were applied for the plane wave expansion of the wave functions and charge density, respectively. For comparison with XPS

experiments, the BEs of the $\mathrm{O} 1$ s core state were calculated within the final-state approximation ${ }^{16}$ 
using density functional theory (DFT) and the PBE exchange-correlation functional. ${ }^{17}$ In addition, to facilitate direct comparison with experimental spectra, the BEs of surface oxygens on and InP were evaluated with respect to that of bulk water oxygens in the corresponding simulation models. The complete screening theory used in this study does not provide a direct energy reference. Therefore, the BE of bulk liquid water was set to the experimental value of $533.8 \mathrm{eV},{ }^{18}$ which in turn was obtained by calibrating against the gas-phase water peak at $535.6 \mathrm{eV}$. The BEs of each oxygen species were then obtained as averages of results computed for 60 snapshots extracted equally from the simulation trajectories. We would like to emphasize that our computational strategy goes beyond the conventional approach by including temperature and water effects in the simulation. This allows for more complete sampling of the configurational space of surface chemical species, thereby representing a more realistic description of solid/liquid interfaces. We note that our core-level binding energy for each chemical species were obtained by averaging results over a large number of configurations, rather by relying on a single configuration of, e.g., hydroxyl hydrogen bonded with a single water molecule. We find that this averaging procedure is necessary, as our calculations show that the binding energy can be sensitive to the local structure of the chemical species, showing a spread as large as $0.5 \mathrm{eV}$ for oxygen computed for different In$\mathrm{OH}-\mathrm{In}$ configurations. Note that, while the absolute BE values may vary depending on the energy reference applied, the $\triangle \mathrm{BEs}$ between difference surface species are expected to be constant under identical calculation/experimental conditions.

In additional to the calculations based on the full hydroxylated interface that is derived from direct first-principles molecular dynamics simulations, we also investigate the $\mathrm{BE}$ of $\mathrm{PO}_{\mathrm{x}}$ species by systematically generating oxygen adsorbed surface structures based on the InP $(001)-\delta(2 \times 4)$ mixed dimer structures, where oxygen concentrations were varied from 1 per $\delta(2 \times 4)$ to 4 per 
$\delta(2 \times 4)$. For the BE calculations of $\mathrm{P}$ species, we adjusted theoretical $\mathrm{P} 2 \mathrm{p} 3 / 2 \mathrm{BE}$ of crystalline InP to our experimentally observed peak position of $128.8 \mathrm{eV}$, together with a spin-orbit splitting value of $0.84 \mathrm{eV}$. The simulated $\mathrm{BE}$ in the present study represents the $\mathrm{H}_{2} \mathrm{O} / \mathrm{InP}(001)$ interface under higher-than-conventional $\mathrm{H}_{2} \mathrm{O}$ coverage conditions. While the coverage dependent $\mathrm{BE}$ shift is out of the scope of the present study, the general trend in terms of the BE (or $\triangle \mathrm{BE}$ ) values of surface species is coverage-independent.
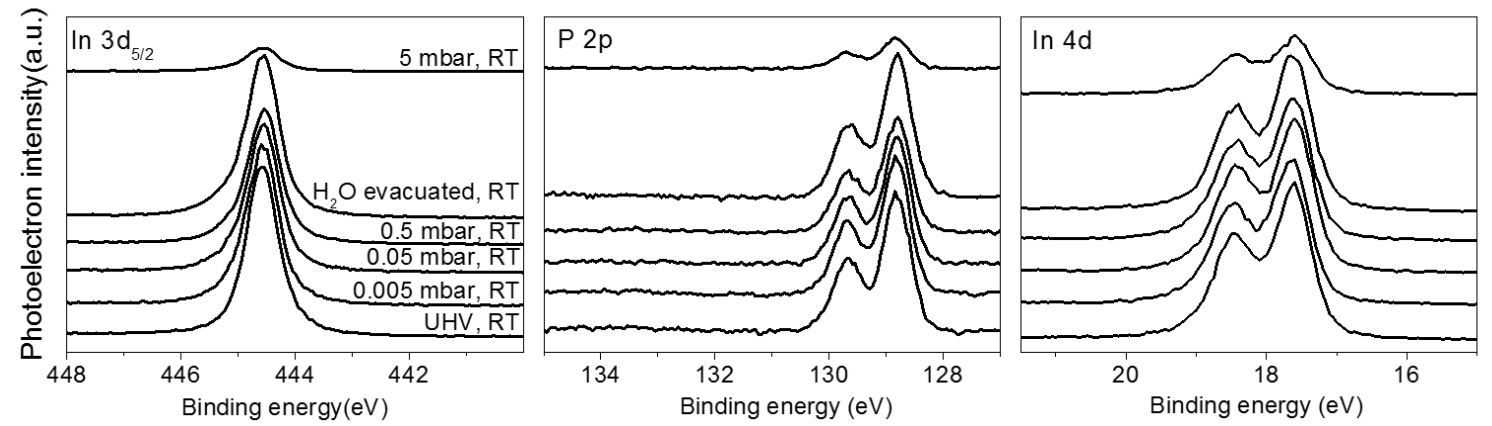

Figure S1 Evolutions of In ( $3 \mathrm{~d}_{5 / 2}$ and $\left.4 \mathrm{~d}\right)$ and $\mathrm{P} 2 \mathrm{p}$ photoemission spectra under different water vapor pressures and after evacuation at RT. The spectra were stacked along y-axis.
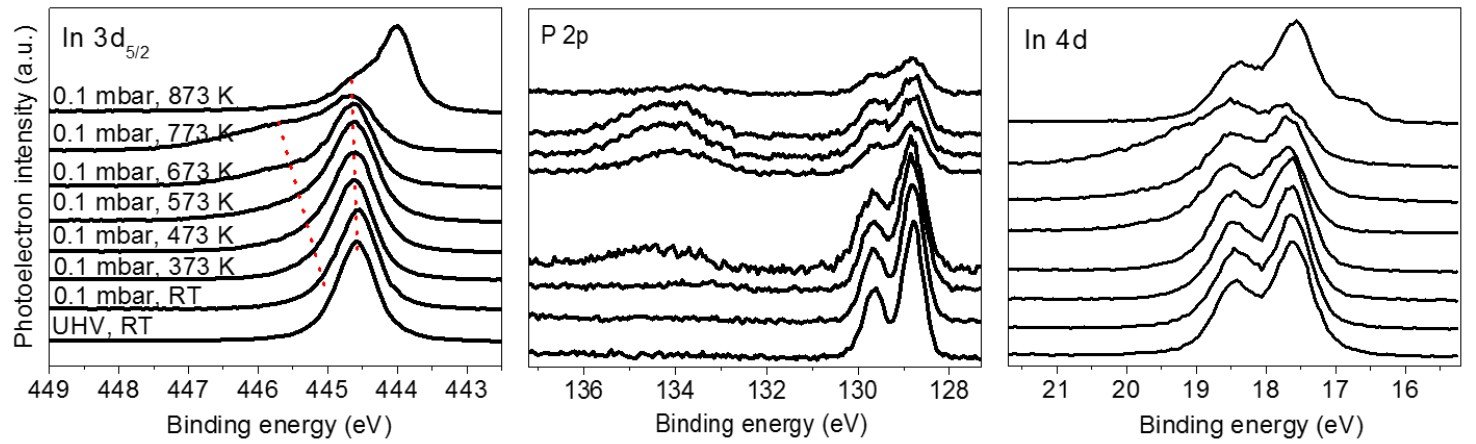

Figure S2 Evolutions of In ( $3 \mathrm{~d}_{5 / 2}$ and $\left.4 \mathrm{~d}\right)$ and $\mathrm{P} 2 \mathrm{p}$ photoemission spectra under different temperatures in 0.1 mbar of $\mathrm{H}_{2} \mathrm{O}$. The spectra were stacked along y-axis. 

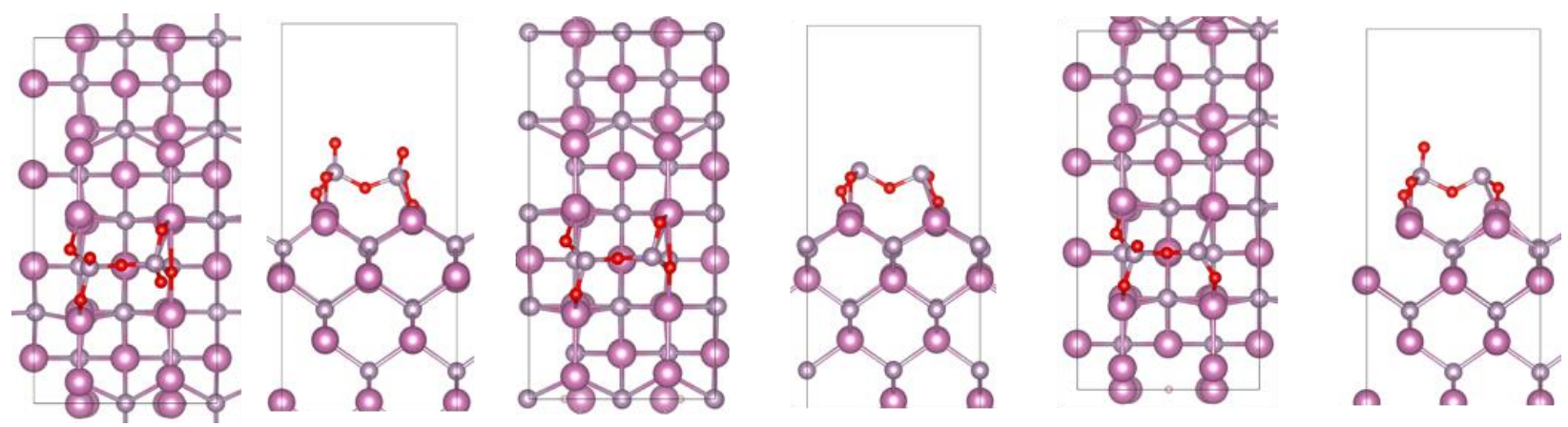

Figure S3 Representative examples of $\mathrm{PO}_{\mathrm{x}}$ configurations. The red, pink and gray balls represent oxygen, In and $\mathrm{P}$ atoms, respectively.

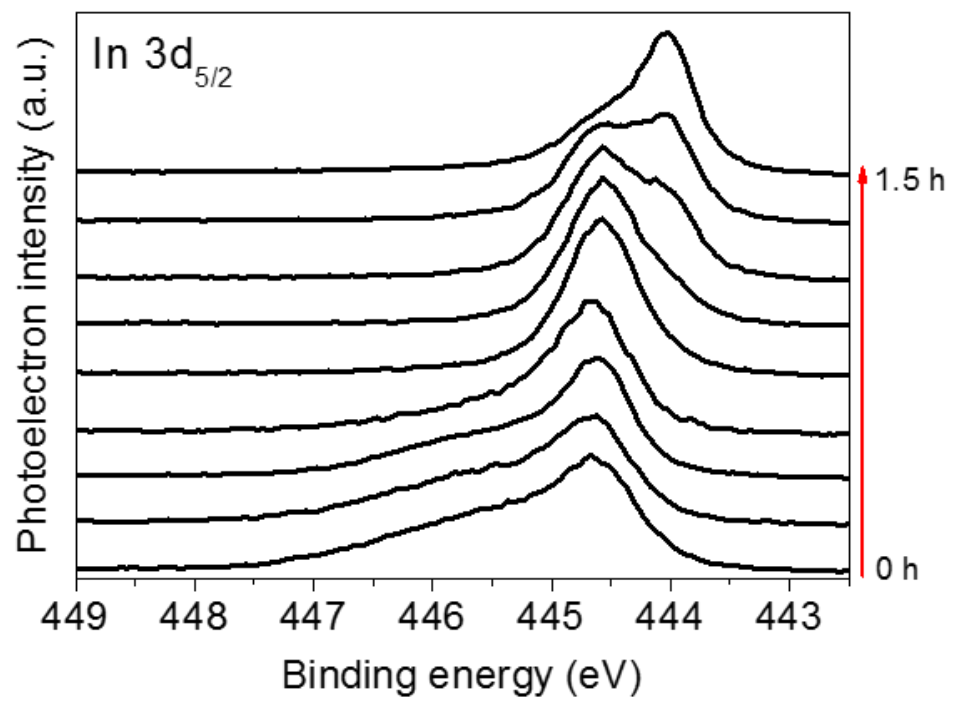

Figure S4 Evolution of In $3 \mathrm{~d}_{5 / 2}$ at $873 \mathrm{~K}$ in 1.5 hours (continuous recording). The dynamic spectral changes suggest desorption of surface oxides/hydroxides species at $873 \mathrm{~K}$ and the breakage of In$\mathrm{P}$ bond. The surface was finally covered by In in its metallic phase. The spectra were stacked along $\mathrm{y}$-axis. 

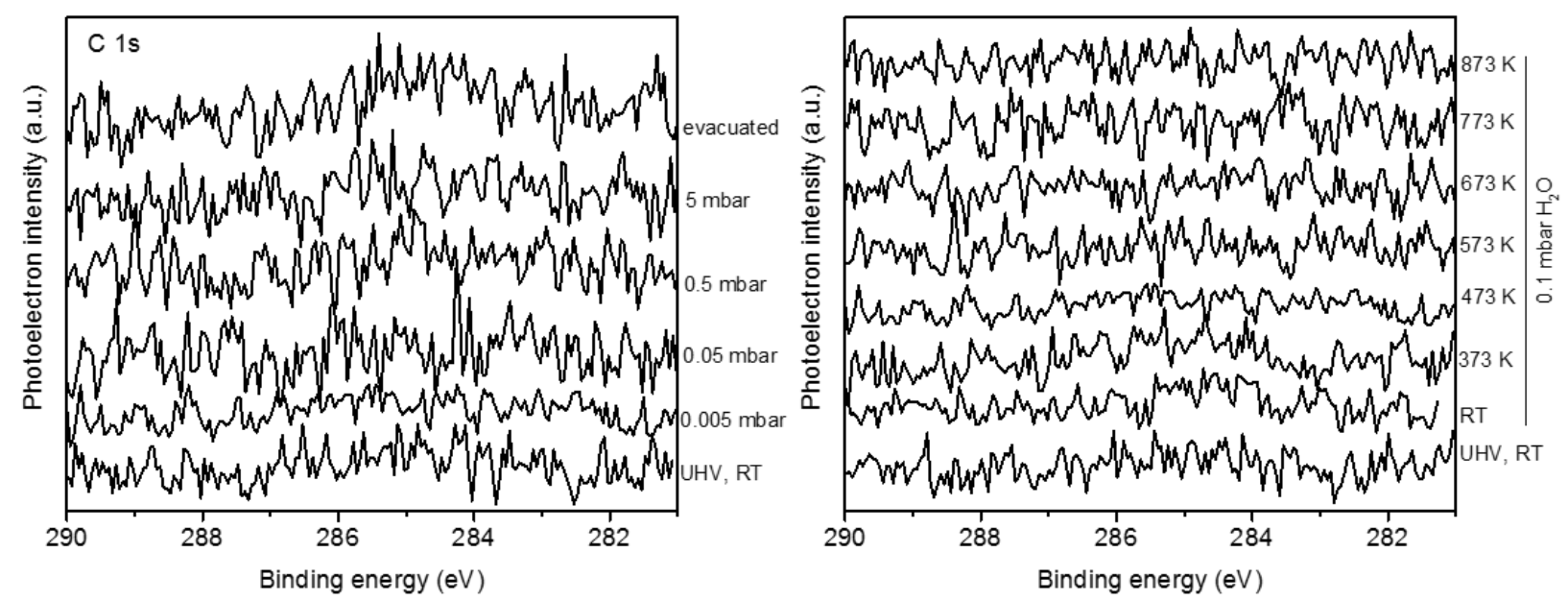

Figure S5 Photoemission spectra of C 1s under different conditions.

\section{Reference}

(1) Zhang, X.; Ptasinska, S. Dissociative Adsorption of Water on an $\mathrm{H}_{2} \mathrm{O} / \mathrm{GaAs}(100)$ Interface: In Situ Near-Ambient Pressure XPS Studies. J. Phys. Chem. C 2014, 118, 4259-4266.

(2) Zhang, X.; Lamere, E.; Liu, X., Y.; Furdyna, J. K.; Ptasinska, S. Interface Chemistry of $\mathrm{H}_{2} \mathrm{O}$ on GaAs Nanowires Probed by Near Ambient Pressure X-ray Photoelectron Spectroscopy. Chem. Phys. Lett. 2014, 605-606, 51-55.

(3) Chen, G.; Visbeck, S. B.; Law, D. C.; Hicks, R. F. Structure-Sensitive Oxidation of the Indium Phosphide (001) Surface. J. Appl. Phys. 2002, 91, 9362-9367.

(4) Streubel, P.; Peisert, H.; Hesse, R.; Chasse, T.; Szargan, R. Chemical Bonding Studies on UV/OzoneTreated and $\left(\mathrm{NH}_{4}\right)_{2} \mathrm{~S}$-Treated InP(001) Surfaces by X-Ray Photoelectron-Spectroscopy and X-Ray-Induced Auger-Electron Spectroscopy. Surf. Interf. Anal. 1995, 23, 581-588.

(5) Franke, R.; Chasse, T.; Streubel, P.; Meisel, A. Auger Parameters and Relaxation Energies of Phosphorus in Solid Compounds. J. Electron Spectros. Relat. Phenomena. 1991, 56, 381-388.

(6) Hollinger, G.; Bergignat, E.; Joseph, J.; Robach, Y. On the Nature of Oxides on InP Surfaces. J. Vac. Sci. Technol. A Vac. Surf. Films 1985, 3, 2082-2088.

(7) Patanen, M.; Aksela, S.; Urpelainen, S.; Kantia, T.; Heinasmaki, S.; Aksela, H. Free Atom $4 \mathrm{f}$ Photoelectron Spectra of $\mathrm{Au}, \mathrm{Pb}$, and Bi. J. Electron Spectros Relat Phenomena. 2011, 183, 59-63.

(8) Sung, M. M.; Lee, S. H.; Lee, S. M.; Marton, D.; Perry, S. S.; Rabalais, J. W. Composition and Morphology of InP (100) Surfaces as a Function of Low Energy $\mathrm{Ar}^{+}$Bombardment and Annealing. Surf. Sci. 1997, 382, 147-153.

(9) Feurprier, Y.; Cardinaud, C.; Turban, G. X-Ray Photoelectron Spectroscopy Damage Characterization of Reactively Ion Etched InP in $\mathrm{CH}_{4}-\mathrm{H}_{2}$ Plasmas. J. Vac. Sci. Technol. B 1998, 16, 1823-1832.

(10) Hu, S. Z.; Scudiero, L.; Ha, S. Electronic Effect on Oxidation of Formic Acid on Supported Pd-Cu Bimetallic Surface. Electrochimica Acta 2012, 83, 354-358.

(11) Ketteler, G.; Ogletree, D. F.; Bluhm, H.; Liu, H. J.; Hebenstreit, E. L. D.; Salmeron, M. In Situ Spectroscopic Study of the Oxidation and Reduction of Pd(111). J. Am. Chem. Soc. 2005, 127, 1826918273. 
(12) Powell, C. J.; Jablonski, A.: Nist Standard Reference Database 71: NIST Electron Inelastic Mean Free Path Database: Version 1.2; National Institute of Standards and Technology: Gaithersburg, Maryland, USA, 2010.

(13) Wood, B. C.; Schwegler, E.; Choi, W. I.; Ogitsu, T. Hydrogen-Bond Dynamics of Water at the Interface with Inp/Gap(001) and the Implications for Photoelectrochemistry. J. Am. Chem. Soc. 2013, 135, 15774-15783.

(14) Wood, B. C.; Ogitsu, T.; Schwegler, E. Local Structural Models of Complex Oxygen-and HydroxylRich GaP/InP (001) Surfaces. J. Chem. Phys. 2012, 136, 064705-064715.

(15) Giannozzi, P.; Baroni, S.; Bonini, N.; Calandra, M.; Car, R.; Cavazzoni, C.; Ceresoli, D.; Chiarotti, G. L.; Cococcioni, M.; Dabo, I. et al. Quantum Espresso: A Modular and Open-Source Software Project for Quantum Simulations of Materials. J. Phys. Condes. Matter 2009, 21, 395502.

(16) Pehlke, E.; Scheffler, M. Evidence for Site-Sensitive Screening of Core Holes at the Si and Ge (001) Surface. Phys. Rev. Lett. 1993, 71, 2338-2341.

(17) Perdew, J. P.; Burke, K.; Ernzerhof, M. Generalized Gradient Approximation Made Simple. Phys. Rev. Lett. 1996, 77, 3865-3868.

(18) Winter, B.; Aziz, E. F.; Hergenhahn, U.; Faubel, M.; Hertel, I. V. Hydrogen Bonds in Liquid Water Studied by Photoelectron Spectroscopy. J. Chem. Phys. 2007, 126, 6. 